\title{
Simulation of stray grain formation in Ni-base single crystal turbine blades fabricated by HRS and LMC techniques
}

\author{
Ya-feng Li, *Lin Liu, Tai-wen Huang, Miao Huo, Jun-sheng He, Jun Zhang, and Heng-zhi Fu \\ State Key Laboratory of Solidification Processing, Northwestern Polytechnical University, Xi'an 710072, China
}

\begin{abstract}
The simulation models of the thermal and macrostructural evolutions during directional solidification of Ni-base single crystal (SX) turbine blades under high rate solidification (HRS) and liquid metal cooling (LMC) have been constructed using ProCAST software, coupled with a 3D Cellular Automaton Finite Element (CAFE) model. The models were used to investigate the tendencies of stray grain (SG) formation in the platform region of turbine blades fabricated by HRS and LMC techniques. The results reveal that the LMC technique can prohibit SG formation by smoothing the concaved isotherm and in turn alleviating the undercooling in the platform ends to let the dendrites fill up the undercooled zone before SG nucleation. The simulation results agreed well with the experimental results, indicating that these models could be used to analyze the macrostructural evolution or to optimize process parameters to suppress SG formation. Using these models, the critical withdrawal rate for casting SX turbine blades without SG formation were determined to be around $75 \mu \mathrm{m} \cdot \mathrm{s}^{-1}$ and $100 \mu \mathrm{m} \cdot \mathrm{s}^{-1}$ for HRS and LMC respectively, suggesting that LMC can be used as an efficient technique in fabricating SX turbine blades without any SG defect formation.
\end{abstract}

Key words: thermal profile; macrostructural evolution; simulation; HRS; LMC; stray grain
CLC numbers: TG146.1 5
Document code: $\mathrm{A}$
Article ID: 1672-6421(2017)02-075-02

$\mathrm{T}$ he superior mechanical properties of Ni-base single crystal (SX) turbine blades are mainly attributed to the elimination of the grain boundaries and the arrangement of the best creep resistance orientation [001] to bear the main stress of the turbine blade in service $^{[1]}$. The formation of stray grains (SGs) during directional solidification process, however, introduces new boundaries to contaminate the perfection of SX, leading to a sharp decrease of the mechanical properties. To inhibit such defect occurrence, investigations have been done aimed to fully understand the locations, the mechanism and the influencing factors of SG formation. It has been determined that these defect grains usually form around re-entrant sections, like shrouds and platforms, and are resulted from the locally changed solidification conditions. Vehn et al. ${ }^{[2]}$ depicted that SG nucleated in a thermally undercooled zone in the platform ends caused by macroscopically curved

\section{*Lin Liu}

Male, born in 1956, Ph.D, Professor. His research and teaching interests mainly focus on the microstructure and properties of cast superalloys, theory and application of directional solidification, electromagnetic confinement and shaping of metallic materials.

E-mail: linliu@nwpu.edu.cn

Received: 2016-10-09; Accepted: 2017-01-06 liquidus isotherm, which is confirmed by $\mathrm{Li}$ et al. ${ }^{[3]}$ through characterizing SG morphologies in multiscale levels. Based on several simplified assumptions, Bussac et al. ${ }^{[4]}$ derived a theoretical model to predict the dependence of SG formation on different factors, such as withdrawal rate ${ }^{[5-7]}$, the inclination angle of isotherms ${ }^{[5]}$ and the platform dimension ${ }^{[6]}$. Using alloys with a large nucleation undercooling can suppress SG formation ${ }^{[8,9]}$. As for different directional solidification techniques [high rate solidification (HRS) and liquid metal cooling (LMC)] on SG formation, Lu et al. ${ }^{[10]}$ and Cao et al. ${ }^{[11]}$ investigated SG formation in the platform region of a dummy turbine blade and found that withdrawal rate can be set up to $8 \mathrm{~mm} \cdot \mathrm{min}^{-1}$ in the $\mathrm{LMC}$ furnace to fabricate SX blades without SG formation; by comparing the thermal profile in HRS and LMC conditions, Tang et al. ${ }^{[12]}$ and Guo et al. ${ }^{[13]}$ confirmed that the LMC technique could largely increase the critical withdrawal rate in fabricating SXs without SG formation in comparison to HRS technique. Based on previous studies, this work investigated the SG formation tendency in an aero turbine blade fabricated by HRS and LMC techniques aiming to assess the advantage of LMC technique on SG formation. The experimental and simulated macrostructures were illustrated followed 
by the orientation analysis to evaluate the misorientation in grain boundary (GB) areas. Local thermal and macrostructural evolution in the platform region were simulated afterwards to explain the SG formation by analyzing the cooling process and the macrostructural evolution in the platform region. Critical withdrawal rates for the turbine blades with no SG formation in both HRS and LMC settings were finally revealed as well.

\section{Experimental and simulation procedure}

SX turbine blades (Fig. 1a) were fabricated in a modified Bridgman furnace, capable of operating both in HRS and LMC modes by emptying or filling up the cooling container with Gallium-Indium-Tin (Ga-In-Sn) liquid metal (Fig. 1b). The commercial SX superalloy DD403 with the composition (wt.\%): $6.5 \mathrm{Cr}, 5.0 \mathrm{Co}, 3.8 \mathrm{Mo}, 5.2 \mathrm{~W}, 5.9 \mathrm{Al}, 2.1 \mathrm{Ti}$ and Ni the balance was used, and the liquidus and solidus of the alloy are
$1,371{ }^{\circ} \mathrm{C}$ and $1,324{ }^{\circ} \mathrm{C}$, respectively, measured by differential thermal analysis (DTA). During directional solidification, the furnace chamber was first evacuated to a partial pressure of approximately $10^{-2} \mathrm{~Pa}$. The ceramic mold was then preheated to $1,550{ }^{\circ} \mathrm{C}$ by graphite heating elements. After the ingot was melted, the liquid metal at $1,500{ }^{\circ} \mathrm{C}$ was poured into the mold cavity and held for $10 \mathrm{~min}$. The ceramic mold was withdrawn out of the heating furnace at a predetermined rate ranging from $50 \mu \mathrm{m} . \mathrm{s}^{-1}$ to $200 \mu \mathrm{m} . \mathrm{s}^{-1}$. Following the casting process, the platforms were sectioned and etched for metallographic examination using a solution of $10 \mathrm{~mL} \mathrm{HNO}_{3}$ : $20 \mathrm{~mL} \mathrm{HF}$ : 30 $\mathrm{mL} \mathrm{C}_{3} \mathrm{H}_{8} \mathrm{O}_{3}$. The dendritic morphologies were observed using an optical microscope (DM-4000M; Leica, Berlin, Germany). EBSD measurements of the crystallographic orientation were performed using a step size of $30 \mu \mathrm{m}$ under a condition of accelerating voltage of $20 \mathrm{kV}$ and a probe current of $4 \mathrm{nA}$ in a scanning electron microscope (SEM) (TESCAN VEGA, TESCAN, Czech Republic) equipped with HKL system and Channel 5 analysis software (Oxford instruments, Oxford, UK). (a)

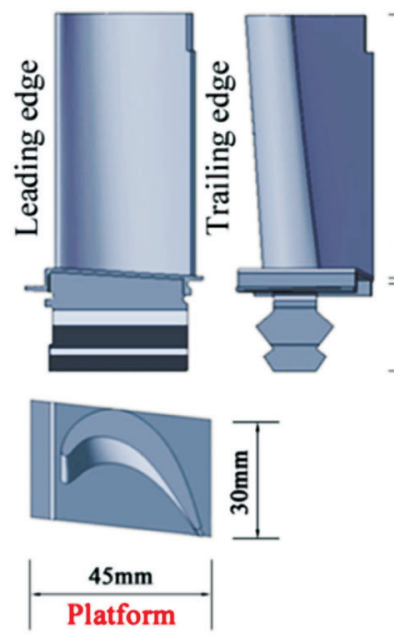

(b)

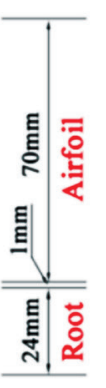

(ans

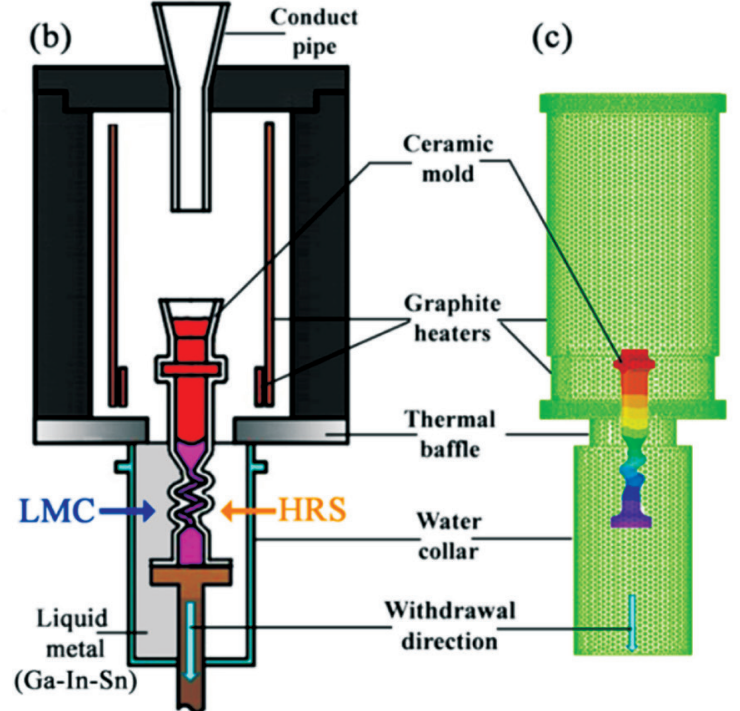

(c)

\begin{abstract}
Fig. 1: Schematic of turbine blade (a), modified Bridgman furnace (b), FE mesh model of the furnace (c) and parameters used in study (d) (Note: the heat conductivity of the metal $\left(\lambda_{\text {Metal }}\right)$ and the interfacial heat transfer coefficient between metal and mold $\left(h_{\mathrm{M} / \mathrm{M}}\right)$ are temperature-dependent, as shown in Table 1)
\end{abstract}

Thermal profiles and macrostructural evolutions were simulated using a 3D finite element analysis software ProCAST (Calcom ESI, Lausanne-EPFL, Switzerland), coupled with a Cellular Automaton Finite Element (CAFE) module. Finite element (FE) mesh models of the turbine blade, the spiral grain selector and the ceramic shell were constructed into 3D mesh. Considering the computational efficiency, the HRS/LMC furnace was constructed into a 2D mesh enclosure including the heating zone (HZ), the cooling zone (CZ) and the thermal baffle (Fig. 1c). The simulation initiated at the state after the holding process; therefore the heating zone was set at the initial temperature of $1,550{ }^{\circ} \mathrm{C}$ and the cooling zone was set to $150{ }^{\circ} \mathrm{C}$ for HRS case and $25^{\circ} \mathrm{C}$ for LMC case (Fig. 1d). During the withdrawal process with HRS, radiation heat transfer between the mold surface and $\mathrm{HZ} / \mathrm{CZ}$ was calculated by a radiation algorithm taking into account reflections, obstructions and shadowing effects. The melt temperature was determined by calculating the heat conduction through the mold shell, the heat transfer between mold and melt and the heat conduction in the melt itself using the parameters as illustrated in Fig. 1d and Table 1. For LMC solidification, the radiation heat transfer was replaced by convective heat transfer once the mold was immersed into the liquid metal. Since the fluid flow of the liquid metal bath cannot be explicitly modeled, an incorporated high heat-transfer coefficient $\left(h_{\mathrm{LMC}}=4000\right)$ was applied to calculate the temperature of the mold surface by convective heat-transfer calculation. The calculated thermal profiles were then imported into CAFE module to predict the grain structure in HRS and LMC process within the consideration of the heterogeneous nucleation, the crystallographic anisotropy of grains and the growth kinetics of dendrite tips. The growth 
Table 1: Thermophysical properties of materials used in simulations

\begin{tabular}{|c|c|c|c|c|}
\hline Material & $\begin{array}{l}\text { Density } \\
\left(\mathbf{k g} \cdot \mathbf{m}^{-3}\right)\end{array}$ & $\begin{array}{c}\text { Thermal } \\
\text { conductivity } \\
\left(\mathrm{W} \cdot \mathrm{m}^{-1} \cdot{ }^{\circ} \mathrm{C}^{-1}\right)\end{array}$ & $\begin{array}{c}\text { Latent } \\
\text { heat } \\
\left(\mathbf{k J} \cdot \mathbf{k g}^{-1}\right)\end{array}$ & $\begin{array}{c}\text { Heat } \\
\text { capacity } \\
\left(\mathrm{kJ}^{\mathrm{J}} \cdot \mathrm{kg}^{-1} \cdot{ }^{\circ} \mathrm{C}^{-1}\right)\end{array}$ \\
\hline DD403 & \multicolumn{2}{|c|}{ Temperature-depended, Table 2} & \multicolumn{2}{|c|}{ Enthalpy based, Table 2} \\
\hline Mold $^{[17]}$ & 2,750 & 2.5 & - & 1 \\
\hline
\end{tabular}

Table 2: Temperature-dependent thermophysical properties of alloy and heat transfer coefficients between interfaces of mold and metal

\begin{tabular}{ccccc}
$\begin{array}{c}\text { Temperature } \\
\left({ }^{\circ} \mathbf{C}\right)\end{array}$ & $\begin{array}{c}\boldsymbol{\rho}_{\text {Metal }} \\
\left(\mathbf{k g} \cdot \mathbf{m}^{-3}\right)\end{array}$ & $\begin{array}{c}\lambda_{\text {Metal }} \\
\left(\mathbf{W} \cdot \mathbf{m}^{-1} \cdot{ }^{\circ} \mathbf{C}^{-1}\right)\end{array}$ & $\begin{array}{c}\text { Enthalpy of } \\
\mathbf{m e t a l} \\
\left(\mathbf{k J} \cdot \mathbf{K g} \mathbf{~}^{-1}\right)\end{array}$ & $\begin{array}{c}\boldsymbol{h}_{\text {MMM }} \\
\left(\mathbf{W} \cdot \mathbf{m}^{-2} \cdot{ }^{\circ} \mathbf{C}^{-1}\right)\end{array}$ \\
\hline 25 & 8.164 & 10.3 & -368.7 & 150 \\
400 & 8016 & 15.3 & 191.1 & 150 \\
1,000 & 7.766 & 23.8 & 159.4 & 190 \\
1,324 & 7505 & 32.8 & 450.1 & 350 \\
1,371 & 7.169 & 30.5 & 700.9 & 750 \\
1,550 & 7016 & 33.4 & 837.3 & 750 \\
& & & &
\end{tabular}

of the dendrite was calculated with the aid of Kurz-GiovanolaTrivedi (KGT) model ${ }^{[14]}$. Since superalloys are multicomponent systems, the extended KGT model suggested by Rappaz et al. ${ }^{[15]}$ was used. The values of the partition coefficient, liquidus slope, and diffusion coefficient of the alloying elements were referred to in a previous study by Kermanpur et al. ${ }^{[16]}$

\section{Results and discussion}

\subsection{HRS and LMC techniques on SG formation}

The tendency of SG formation in the platform region of the turbine blades fabricated using HRS and LMC techniques were investigated by both experiments and simulations (Fig. 2). In HRS case, SG formed in the platform edge and grew into the airfoil section to constitute high angle grain boundary (HAB) of $47^{\circ}$ with the primary grain (Fig. $2 \mathrm{~b}, \mathrm{~b} 1$ ). In the LMC case, however, the platform edge was filled up with the dendrites inherited from the primary grain and the EBSD measured misorientation from Zone 1 to 3 is less than $5^{\circ}$ (Fig. 2d, d1). The

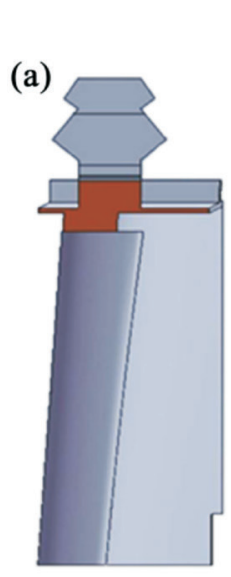

HRS V $=100 \mu \mathrm{m} \cdot \mathrm{s}^{-1}$
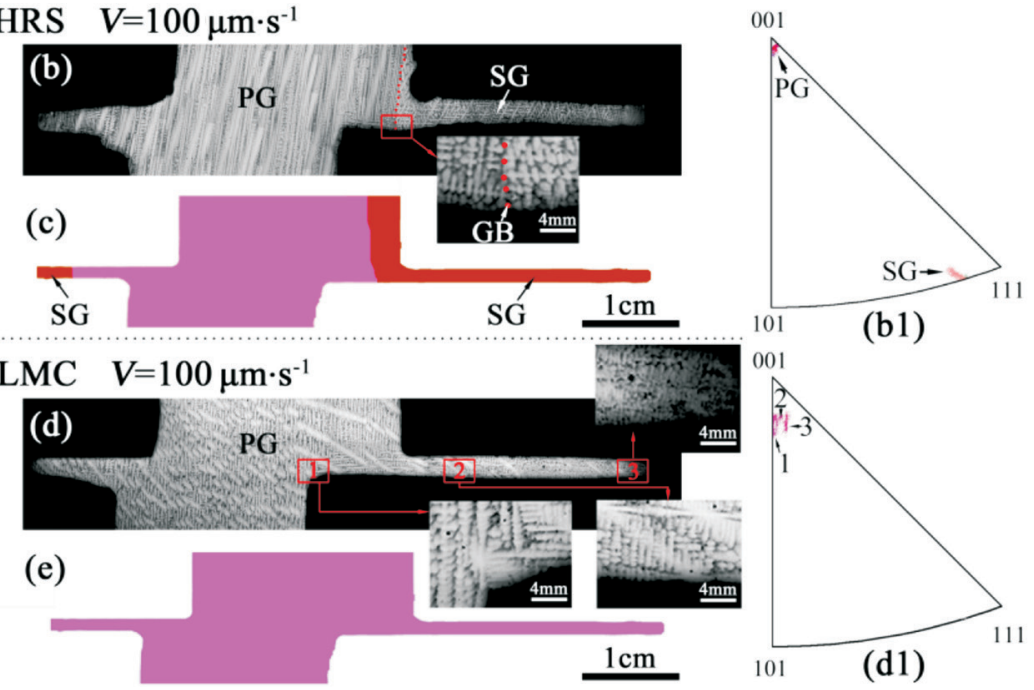

Fig. 2: Schematic of cutting site of sample (a), dendritic morphologies from experiment (b), (d), and macrostructures from CAFE simulations in HRS (c) and LMC (e) cases; inverse pole figures in (b1) and (d1) showing the orientations of red-line framed areas in (b), (d) (PG: primary grain; SG: stray grain; GB: grain boundary)

simulations showed similar results with the experiments (Fig. $2 \mathrm{c}, \mathrm{e})$. It is well known that SG formation in the platform region is a nucleation-growth process initially happening in a thermal undercooled zone in the platform ends. The formation of this undercooling zone is attributed to the radiation heat transfer associated with the cantilever configuration of the platform. Thermal evolution in the local platform region, therefore, is a crucial factor that dominates SG formation.

Thermal evolutions were modeled to account for the aforementioned different SG formation phenomena in HRS and LMC cases (Fig. 3). In the HRS case, the isotherm shows a concave feature as liquidus advanced to the position 10 $\mathrm{mm}$ away from the platform region (Fig. 3a0). The concaved liquidus isotherm reaches the platform section at the trailing edge first (Fig. 3a1). At this time, the isolated undercooling zone has already formed in the platform ends because of the radiation heat transfer in these areas. This zone may breed a new embryo to form SG if the branches from the primary crystal cannot reach these platform ends. The isolated undercooling zone then enlarges and connects with the isotherm in the trailing edge as the solidification continues (Fig. 3a). The isotherm advances into the platform body thereafter from the platform end in trailing edge to the end in the leading edge (Fig. 3a3). Due to the thermal radiation related hot spot zone existing in the sunken area of the airfoil, the thermal contour leaves the platform region at last from this spot which is correspondingly the last solidification area (Fig. 3a4, a5). In the LMC case, the isotherm shows a planar feature (Fig. 3b0) and reaches the platform 


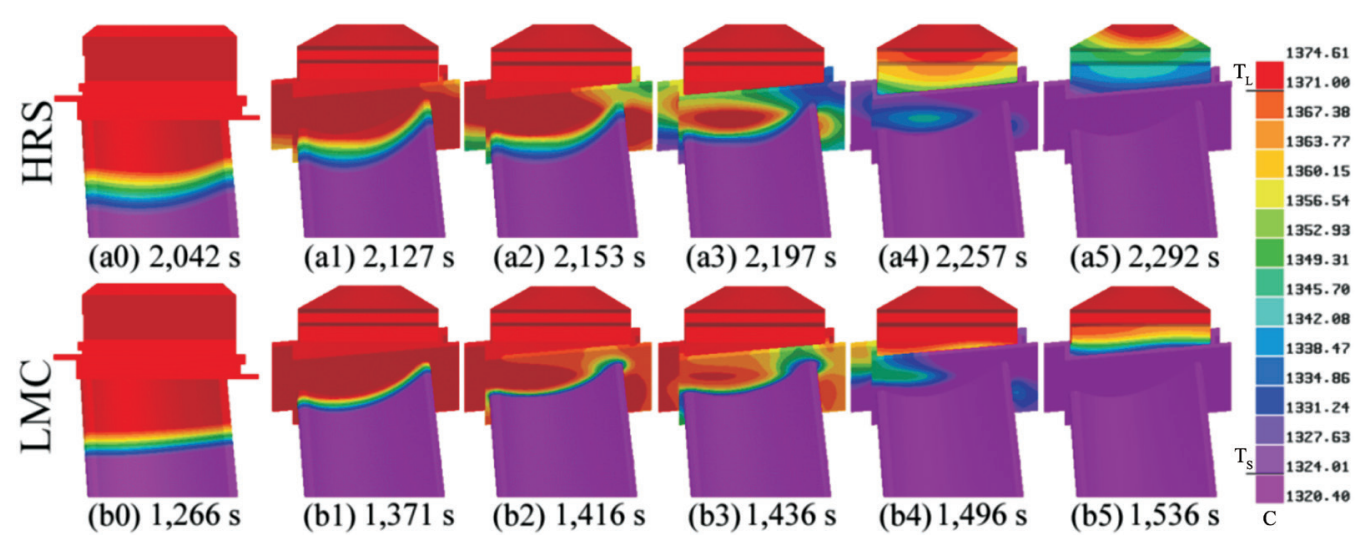

Fig. 3: Thermal contours of solidifying blades at different times fabricated using HRS and LMC technique

region before the isolated undercooling zone forms (Fig. 3b1). The advancement of the isotherm shows a similar pattern as that in the HRS case (Fig. 3b2-b5), except that the hot spot zone moved out of the sunken area a little bit because of the enhanced efficiency of heat conduction in the airfoil section.

The macrostructural evolution and SG formation under these thermal environments in the HRS and LMC cases were investigated by means of CAFE simulations using the thermal simulation data as inputs. In the HRS situation, the solidification front shows a concave feature (Fig. 4a0). The macrostructure then spreads into the platform along with the thermal evolution. During this time, SG forms in the platform end at the trailing edge (Fig. 4a1) and then competes with the primary grain afterwards (Fig. 4a2). As the solidification proceeds, another SG forms in the area around the end of the leading edge and finally constitutes HABs with the primary grain (Fig. 4a3-a5). In the LMC case, the solidification front is comparatively planar (Fig. $4 \mathrm{~b} 0$ ). The primary grain reaches and gets into the platform region along the advancement of the isotherm, and finally fills up the whole platform region without SG formation (Fig. 4b1-b5). This could be ascribed to the combined effect of (1) the primary grain getting into the platform early and (2) the small undercooling in the platform ends, offering enough time for the primary grain to spread and grow.

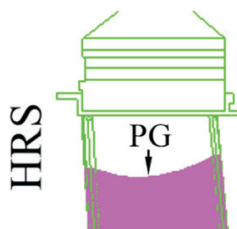

(a0)

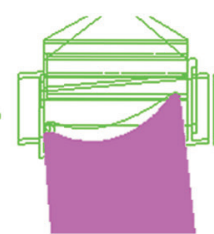

(a1)

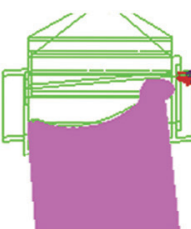

(a2)

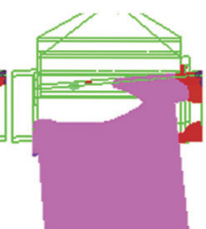

(a3)

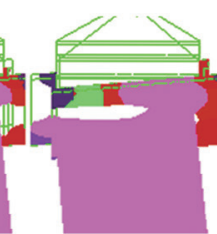

(a4)

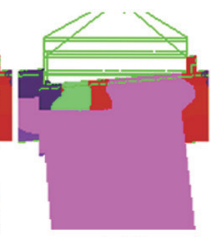

(a5) $t$

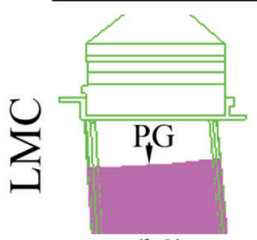

(b0)

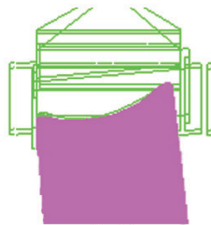

(b1)

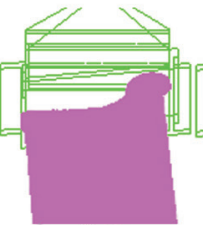

(b2)

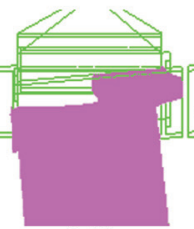

(b3)

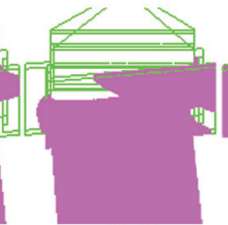

(b4)

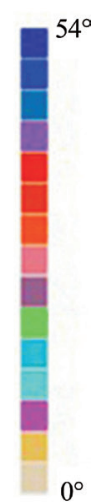

(b5)

Fig. 4: CAFE simulations of the macrostructural evolutions in the solidifying blades fabricated using HRS and LMC techniques

\subsection{Critical withdrawal rate of SG formation in HRS and LMC techniques}

The critical withdrawal rate of SG formation in HRS and LMC techniques are investigated by simulation. In the HRS case, the critical withdrawal rate is between $50 \mu \mathrm{m} \cdot \mathrm{s}^{-1}$ and $75 \mu \mathrm{m} \cdot \mathrm{s}^{-1}$ (Fig. 5a5c); while, by using the LMC technique, the critical withdrawal rate is increased to the range between $100 \mu \mathrm{m} \cdot \mathrm{s}^{-1}$ and $125 \mu \mathrm{m} \cdot \mathrm{s}^{-}$ ${ }^{1}$ (Fig. 5d-5f). This means that the productive efficiency will nearly be doubled or the cost per blade will almost be halved by using LMC technique in comparison to HRS technique. Besides, it should be noted that the withdrawal rate cannot surpass 150 $\mu \mathrm{m} \cdot \mathrm{s}^{-1}$ in the LMC case because SG nucleation in spiral section (grain selection failure) becomes a major concern (Fig. $5 \mathrm{f}, 5 \mathrm{~g}$ ), if the SG in the platform is further controlled by additional means, such as variation of the withdrawal rate ${ }^{[2]}$, using a graphite heat conductor in the hot spot section ${ }^{[17]}$, bypass technique ${ }^{[2,18]}$ or an inner radiation baffle ${ }^{[19]}$.

\section{Conclusions}

The effects of the HRS and LMC techniques on SG formation in the platform region were investigated by experiment and simulation, and the conclusion can then be drawn as follows:

(1) In comparison to HRS, LMC can effectively control the SG formation by enhancing the heat conduction in the airfoil region and therefore alleviating the undercooling in the platform ends to ease the proneness of SG formation.

(2) The critical withdrawal rate for the LMC technique on SG 
HRS

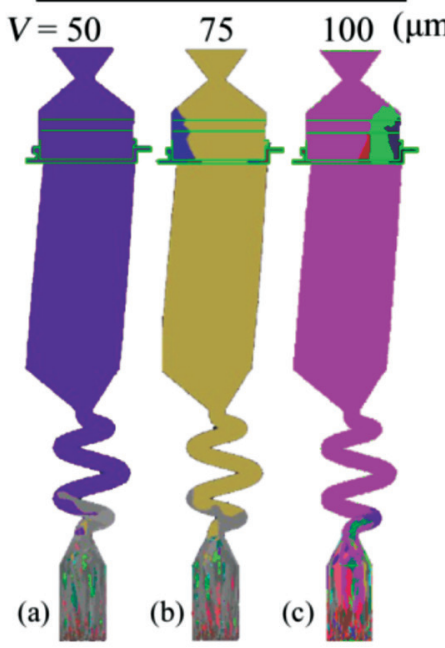

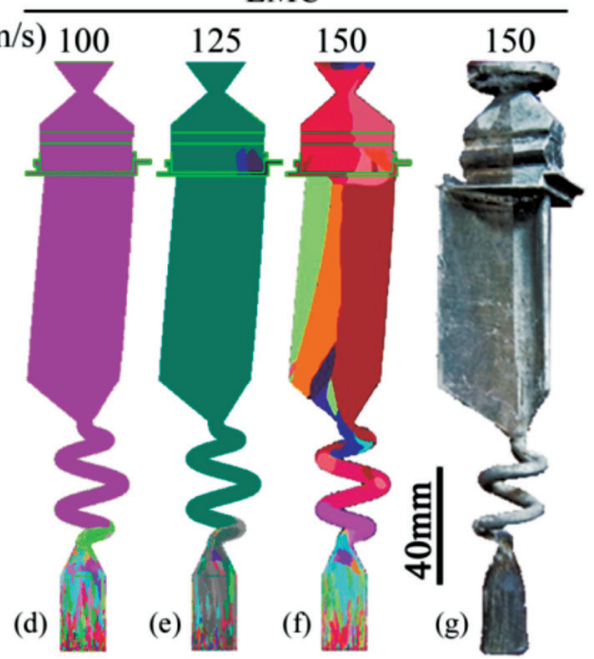

Fig. 5: Macrostructures of turbine blades fabricated using HRS and LMC techniques with different withdrawal rates: (a)-(f) simulation results; (g) experimental result

formation is between $100 \mu \mathrm{m} \cdot \mathrm{s}^{-1}$ and $125 \mu \mathrm{m} \cdot \mathrm{s}^{-1}$ which is higher than that for HRS technique of between $50 \mu \mathrm{m} \cdot \mathrm{s}^{-1}$ and $75 \mu \mathrm{m} \cdot \mathrm{s}^{-1}$.

(3) The withdrawal rate of the directional solidification process cannot be higher than $150 \mu \mathrm{m} \cdot \mathrm{s}^{-1}$, otherwise, grain selection failure will occur.

\section{References}

[1] Reed R C. The Superalloys Fundamental and Applications. Cambridge University Press, Cambridge, UK, 2006: 1-5.

[2] Vehn M M, Dedecke D, Paul U et al. Undercooling related casting defects in single crystal turbine blades. Superalloys1996, TMS, 1996: 471-479.

[3] Li Yafeng, Liu Lin, Huang Taiwen, et al. Multi-scale characterization of stray grain in the platform of nickel-base single crystal turbine blade. Vacuum, 2016, 131: 181-187

[4] Bussac A D and Gandin C A. Prediction of a process window for the investment casting of dendritic single crystals. Materials Science and Engineering A, 1997, 237: 35-42.

[5] Yang X L, Dong H B, Wang W, et al. Microscale simulation of stray grain formation in investment cast turbine blades. Materials Science and Engineering A, 2004, 386: 129-139.

[6] Meng X B, Li J G, Chen Z Q, et al. Effect of platform dimension on the dendrite growth and stray grain formation in a Ni-base single crystal superalloy. Metallurgical and Materials Transaction, 2013, 44A: 1955-1965

[7] Gao Sifeng, Liu Lin, Zhang Jun, et al. Simulation of stray grain formation at the platform during Ni-base single crystal superalloy DD403 casting. China Foundry, 2015, 12: 118-122.

[8] Ma DX, Wu Q, Polaczek AB. Undercoolability of superalloys and solidification defects in single crystal components. Advanced Materials Research, 2011, 278: 114-119.

[9] Zhang Xiaoli, Zhou Yizhou, Jin Tao, et al. Study of the tendency of stray grain formation of $\mathrm{Ni}$-based single crystal superalloys. Acta Metallurgica Sinica, 2012, 48: 1229-1236. (In Chinese)
[10] Lu Yuzhang, Wang Dawei, Zhang Jian, et al. Numerical simulation and experimental observation of single crystal castings processed by liquid metal cooling technique. Foundry, 2009, 58(3): 245-248. (In Chinese)

[11] Cao Liu, Liao Dunming, Lu Yuzhang, et al. Heat transfer model of directional solidification by LMC process for superalloy casting based on finite element method. Metallurgical and Materials Transactions, 2016, 47A: 4640-4647.

[12] Tang Ning, Zhang Hang, Xu Qingyan, et al. Numerical simulation of temperature field and microstructure during directional solidification hollow blades by LMC. Special Casting \& Nonferrous Alloys, 2014, 34(6): 561-565. (In Chinese)

[13] Guo Rufeng, Liu Lin, Li Yafeng, et al. Numerical simulation of temperature field and grain texture during casting single crystal superalloy DD403 with liquid metal cooling. Foundry, 2014, 63(2): 145-151. (In Chinese)

[14] Kurz W, Giovanola B and Trivedi R. Theory of Microstructural development during rapid solidification. Acta Materialia, 1986, 34(5), 823-830.

[15] Rappaz M, David S A, Vitek J M, et al. Analysis of solidification microstructures in Fe-Ni-Cr single-crystal welds. Metallurgical and Materials Transactions, 1990, 21A: 1767-1782.

[16] Kermanpur A, Varahram N, Davami P, et al. Thermal and grainstructure simulation in a land-based turbine blade directionally solidified with the liquid metal cooling process. Metallurgical and Materials Transactions, 2000, 31B: 1293-1304.

[17] Ma D X and Bührig-Polaczek A. Application of a heat conductor technique in the production of single-crystal turbine blades. Metallurgical and Materials Transactions, 2009, 40B: 738-748

[18] Meng X B, Li J G, Zhu S Z, et al. Method of SG inhibition in the platforms with different dimensions during directional solidification of a Ni-base superalloy. Metallurgical and Materials Transactions, 2014, 45A :1230-1236.

[19] Szeliga D, Kubiak K, Sieniawski J. Control of liquidus isotherm shape during solidification of Ni-based superalloy of single crystal platforms. Journal of Materials Processing Technology, 2016, 234: 18-26.

The study was financially supported by the National Key Research and Development Program (2016YFB0701405), the National 973 Program (2011CB610406), National 863 Project (2012AA03A511), the National Natural Science Foundation of China (51171151, 51331005, 51501151, 51631008), the Natural Science Foundation of Shaanxi Province (2014JM6227), the Aeronautical Science Foundation of China (2015ZE53059), and the Fund of the State Key Laboratory of Solidification Processing in NWPU (SKLSP201411). 